\title{
Causas de los conflictos socioambientales en el Perú - 2018
}

\section{Causes of socio-environmental conflicts in Peru - 2018}

Enrique G. Parillo Sosa

eparrillo@ unaj.edu.pe - Universidad Nacional de J uliaca

Carmen E. Zela Pacor

ce.zela@ unaj.edu.pe - Universidad Nacional de J uliaca

Recibido el 16/08/20 | Aceptado el 03/09/20

DOI: https://doi.org/10.47190/nric.v3i1.128

\section{Resumen}

La investigación tuvo como objetivo identificar las causas de los conflictos socioambientales. El método es de alcance descriptivo. Los casos estudiados fueron 5 departamentos con mayor número de conflictos sociales registrados en el año 2018. Para la recolección de datos se aplicó el análisis documental de 12 reportes de la Defensoría del Pueblo del periodo 2018. Producto de la investigación se obtuvo que las causas con mayor frecuencia en los conflictos socioambientales están vinculadas con el incumplimiento de compromisos y convenios con el $29.5 \%$, la afectación a los recursos hídricos con $23 \%$ y el $21.3 \%$ por contaminación ambiental. Se concluye que, según los reportes de la Defensoría del Pueblo en el año 2018, las causas más frecuentes de los conflictos socioambientales son los compromisos arribados entre las partes en conflicto, no se cumplieron, además la contaminación de los recursos naturales (agua y tierra) sigue marcando la conflictividad social en el Perú.

Palabras claves: Contaminación ambiental, conflictos socioambientales, Defensoría del Pueblo, minería.

\begin{abstract}
The research aimed to identify the causes of socio-environmental conflicts. The method is descriptive in scope. The cases studied were 5 departments with the highest number of social conflicts registered in 2018. For data collection, the documentary analysis of 12 reports of the Ombudsman's Office from the 2018 period was applied. As a result of the investigation, it was obtained that the causes with The higher frequency of socio-environmental conflicts is linked to non-compliance with commitments and agreements with $29.5 \%$, the impact on water resources with $23 \%$ and $21.3 \%$ due to environmental contamination. It is concluded that, according to the reports of the Ombudsman's Office in 2018, the most frequent causes of socio-environmental conflicts are the commitments made between the parties in conflict, they were not fulfilled, in addition to the contamination of natural resources (water and land) continues to mark the social conflict in Peru.
\end{abstract}

Keywords: Environmental pollution, socio-environmental conflicts, Ombudsman's Office, mining.

Como citar: Parillo Sosa, E.G. \& Zela-Pacori, C.E. (2020). Causas de los conflictos socioambientales en el Perú - 2018. ÑAWPRISUN - Revista de Investigación Científica, 3(1), 65-70. 


\section{Introducción}

La preocupación por la conflictividad social es una constante desde el Estado peruano. Sin embargo, cabe recordar que el Perú vivió casi veinte años en conflicto armado interno, donde hubo muchas más víctimas mortales que en ciento ochenta y dos años de vida republicana; ni la guerra con Chile generaron tanto sufrimiento y dolor.

Los factores que explican este período de nuestra historia están marcados por la confrontación de los grupos alzados en armas contra el Estado peruano; las brechas sociales subsistentes expresadas en el sofocante centralismo del país; la fuerte gravitación económica, demográfica y simbólica de Lima y la costa en desmedro de los Andes; las divisiones estamentales; un Estado poco legitimado ante la sociedad que lo sentía distante o hasta amenazador; vacíos de poder en amplias zonas del país, fundamentalmente rurales y sobre todo altoandinas; la debilidad de los partidos políticos y una coyuntura económica vulnerable y de persistente crisis (Bedoya, 2014).

Culminado el ciclo del conflicto armado interno, en nuestro país, se fue configurando una nueva etapa que inicialmente estuvo marcada por el reflujo de la conflictividad social. Es a partir de la década de 1990 , durante el gobierno de Fujimori, se implantó un modelo de desarrollo de libre mercado que promovía una apertura comercial y el ingreso de capitales desde el exterior. De hecho, bajo su gobierno, se produjo una apertura a la inversión extranjera, principalmente en el rubro de las industrias extractivas. Debido al contexto de privatizaciones y cambios en las normas de trabajo, los conflictos más recurrentes en esos años fueron laborales, protagonizados básicamente por trabajadores estatales (Flores Unzaga, 2016).

Es en este periodo donde empezaban a crecer notoriamente las concesiones mineras, con posiciones de rechazo de las comunidades hacia proyectos mineros que contravenían sus modos de vida; no obstante, recién en la década siguiente, se harían visibles algunos casos emblemáticos (Barrantes, Tanaka, Vera, \& Pérez-León, 2010; Bedoya, 2014; Mendoza, n.d.). Hacia el final del mandato de Fujimori (1997- 2000) las protestas de mayor importancia fueron de tipo político debido a los indicios de corrupción, al autoritarismo, a las prácticas antidemocráticas del Gobierno y a la pretensión de una segunda reelección (Flores Unzaga, 2016).

A partir del año 2000, la mayor parte de las protestas estuvo centrada, en primer lugar, en cuestionamientos a la gestión de autoridades locales con intentos de remoción de alcaldes y regidores. Se produjeron cambios en la legislación municipal que reorientaron mayores recursos hacia los gobiernos locales (Foncomún), lo que generó mayores disputas por su control, expectativas en la población e inquietudes respecto a las inversiones. Dicha situación se agravó debido a un proceso de desestructuración de las organizaciones sociales y de los partidos políticos durante la década de 1990.
En los siguientes años, el riesgo de este tipo de conflictos se vería incrementado a causa de la distribución del canon minero, tanto que, a partir de 2004, la Defensoría del Pueblo inicia su reporte mensual de conflictos (Bedoya, 2014). Los casos de conflictividad predominantes se relacionaban con enfrentamientos en torno a los gobiernos locales y a las tierras, desde la segunda mitad de esa década hasta la fecha, impulsados por el boom de inversiones mineras y extractivas, los casos vinculados cobran primacía, especialmente los de minería en la zona andina y los de petróleo en la Amazonía (Barrantes et al., 2010; Bury, 2007; Echave et al., 2009; Flores Unzaga, 2016; Pinto, 2009; Tanaka et al., 2007)

Se hacen notorios en la escena nacional algunos casos de oposición a la minería, como Tambogrande (2002), Cerro Quilish (2003- 2004) y Río Blanco (2004-2007), entre otros. De esta forma, la conflictividad socioambiental empieza a posicionarse en la agenda pública (Bedoya, 2014; Echave et al., 2009; Flores Unzaga, 2016; Mendoza, n.d.).

Entre el 2000 y el 2006, años que coinciden con el gobierno de transición del presidente Paniagua y el gobierno de Toledo (2001-2006), se inició un período de reconfiguración de la democracia y, en paralelo, una continuación de las reformas de mercado. En cuanto a la conflictividad social, cobraron importancia las protestas laborales, los conflictos en torno a gobiernos locales (debido a irregularidades y falta de transparencia), los conflictos por posiciones de rechazo a grandes proyectos mineros, además de protestas en contra de las privatizaciones, el TLC (tratado de libre comercio) con Estados Unidos y en defensa del agro.

La gesta de Arequipa o el "Arequipazo", se dio a principios del 2002 y se expresó en una grave revuelta, en el departamento de Arequipa, como oposición al intento del Gobierno de turno de privatizar la empresa generadora de energía eléctrica de Arequipa (Flores Unzaga, 2016).

A partir del 2005, las cifras difundidas por la Defensoría del Pueblo empiezan a dar cuenta de una singular tendencia: los conflictos socioambientales empiezan a dominar la escena social. En adelante, del total de conflictos identificados por esta institución, este tipo de conflictos serían los dominantes hasta la fecha (Bedoya, 2014).

Desde la reinstauración de la democracia a principios del presente siglo. En estos últimos 15 años, tal conflictividad ha sido compleja y variada, habiendo evolucionando no solo en cantidad sino también en su naturaleza y tipo, así como en su ubicación territorial, entre otras variables (Mendoza, n.d.). Estos conflictos desencadenados en los últimos años pusieron en evidencia las serias limitaciones del Estado para afrontarlos, desnudándose su precariedad institucional y su carencia de estrategias en la materia (Flores Unzaga, 2016).

Por ende, el abordaje de los conflictos sociales, es fundamental, especialmente de los que se suscitan en torno a la actividad minera y petrolera en el ciclo 
expansivo de las industrias extractivas. Ello involucra estudiar las causas de los conflictos socioambientales.

\section{Materiales y métodos}

El estudio es de alcance descriptivo, no experimental de corte longitudinal.

Los casos estudiados fueron 5 departamentos del país con mayor frecuencia de conflictividad social reportados por la Defensoría del Pueblo en el año 2018, los cuales fueron; Ancash, Apurímac, Cajamarca, Cusco y Puno.

Se utilizó un instrumento de medición de tipo análisis documental con 9 ítems, se analizó 12 reportes de la Defensoría del Pueblo desde enero a diciembre del año 2018. Finalmente se procesó la información en el paquete estadístico SPSS versión 25.0.

\section{Resultados y discusión}

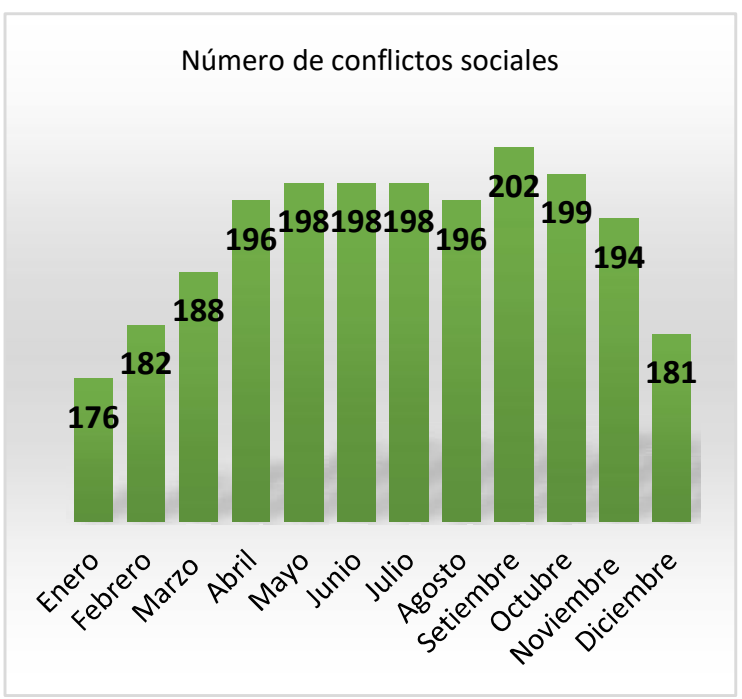

Figura 1. Número de conflictos sociales reportados por la Defensoría del Pueblo en el año 2018

En la figura 1 se muestra el número de conflictos sociales reportados por la Defensoría del Pueblo, en el periodo 2018, en el cual se puede observar que en el mes de setiembre se registró 202 casos de conflictos sociales, el menor número se registró en el mes de enero. El promedio de casos de conflictividad para este año es de 192.

Cuando se analiza el departamento con mayor número de conflictos sociales registrados por la Defensoría del Pueblo en el año 2018 en los meses enero a diciembre se puede apreciar en la Figura 2 , que el departamento de Ancash se encuentra en el primer lugar al ser el departamento con mayor conflictividad en el Perú, siendo el mes de setiembre el más alto con 31 casos registrados. En segundo lugar, se ubica el departamento de Puno, registrando en los meses de mayo, junio y octubre 19 casos de conflictividad social. En tercer lugar, se encuentra el departamento de Apurímac, en el cuarto lugar está Cusco y en el quinto lugar Cajamarca.
Departamentos con mayor numero de conflictos sociales

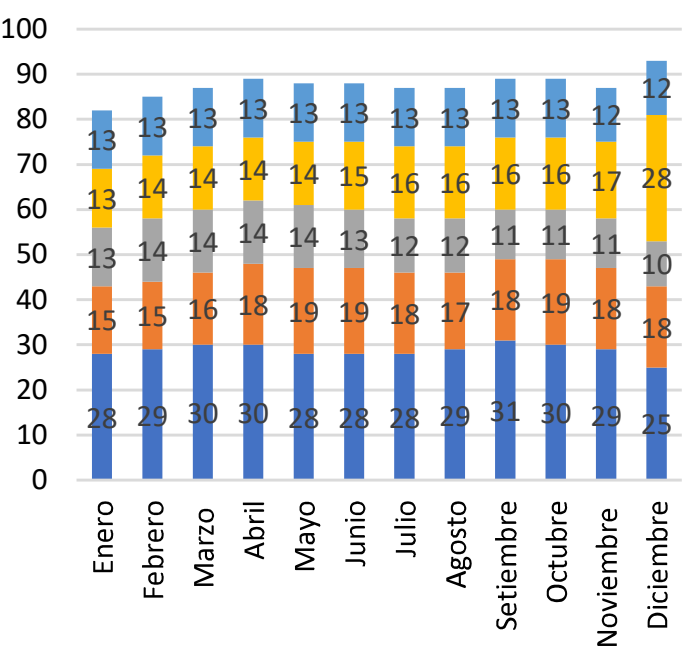

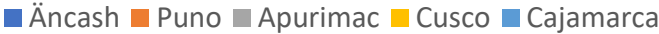

Figura 2. Departamentos con mayor número de conflictos sociales reportados por la Defensoría del Pueblo en el año 2018.

Al observar el tipo de conflicto social registrado por la Defensoría del Pueblo en el año 2018 en los meses enero a diciembre se puede apreciar en la Figura 3, que el tipo de conflicto con mayor frecuencia es el socioambiental, siendo los meses de abril y julio el mayor número de casos con 127, en segundo lugar, el tipo de conflicto social más frecuente para este año son de asuntos de gobierno local, en tercer lugar el de asuntos de gobierno nacional, en cuarto lugar el comunal y con un número menor el territorial.

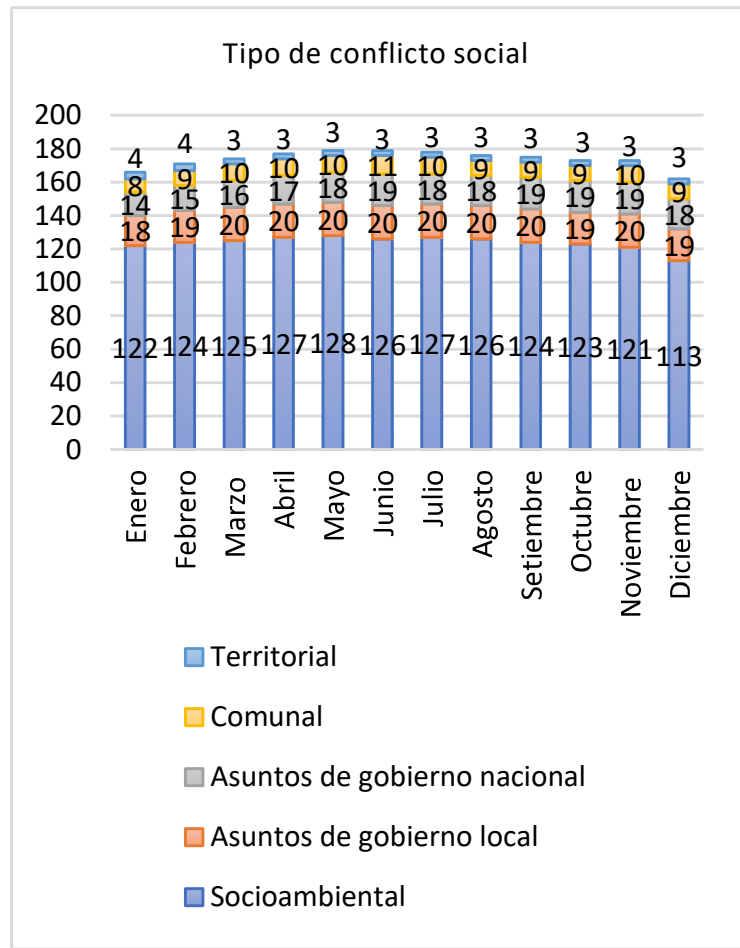

Figura 3. Tipo de conflictos sociales con mayor número reportados por la Defensoría del Pueblo en el año 2018. 
Así mismo, al observar la frecuencia de las causas que impulsan estos conflictos socioambientales se puede apreciar en la figura 4, que predominan el incumplimiento de compromisos y convenios, la afectación a los recursos hídricos, la contaminación ambiental, el temor a la contaminación de recursos naturales, y el acceso y uso a recursos naturales a favor de la empresa minera. En menor porcentaje se encuentra el pedido de remediación de los impactos ambientales, así como efectos negativos a la salud de la población que viven en torno a la empresa minera.

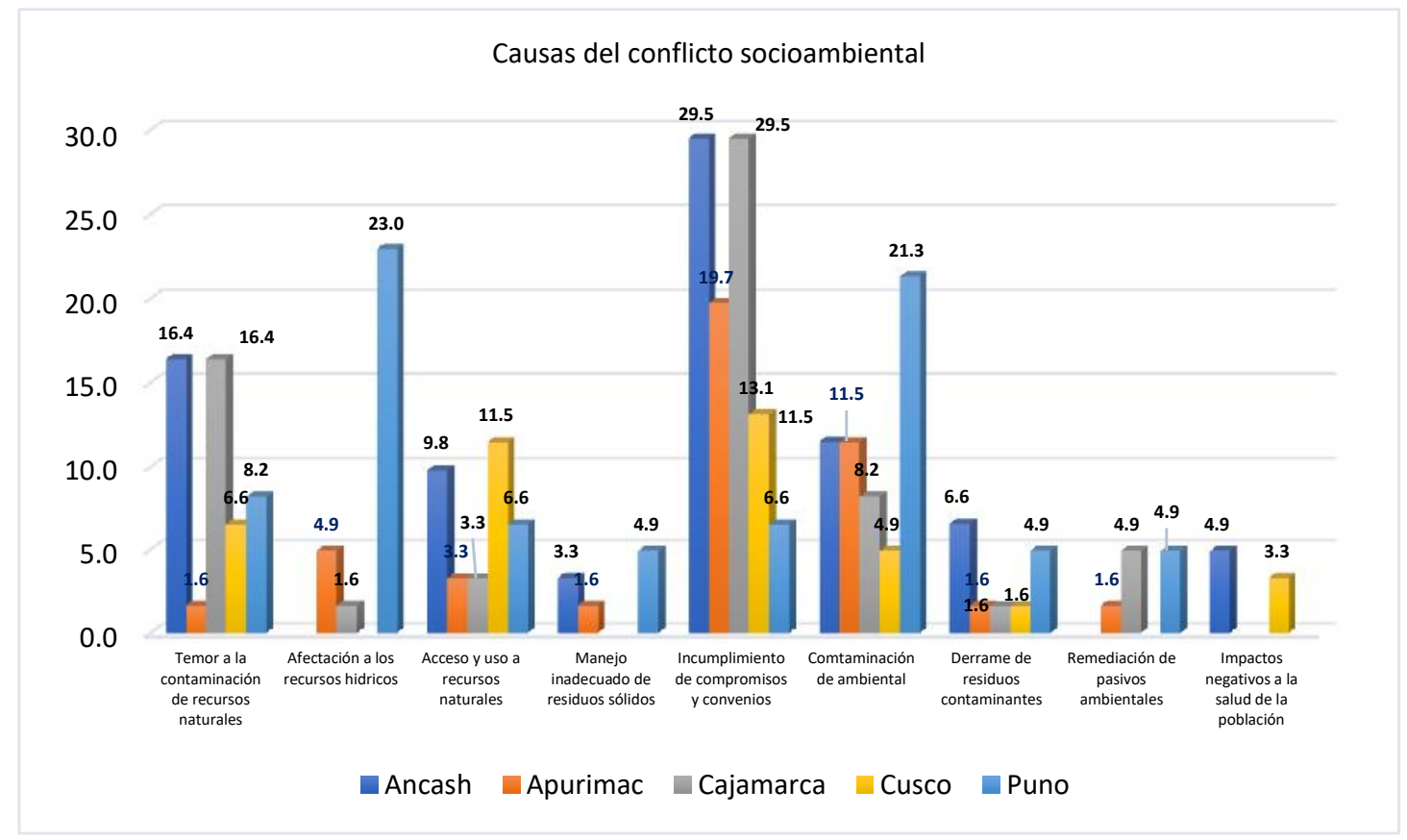

Figura 4. Causas más frecuentes de los conflictos sociales reportados por la Defensoría del Pueblo en el año 2018.

\section{Discusión}

En el Perú, los conflictos socioambientales han crecido en los últimos años de manera más intensa, duradera y con agendas más complejas. Han movilizado cada vez a más personas, las cuales se perciben eventualmente afectadas o excluidas de los beneficios, donde sus cosmovisiones son distintas en relación con el medio ambiente -agua- valor intangible de tierra y agua, desconfianza en la negociación bilateral (Huamaní, Macassi, Alegría, \& Rojas, 2012).

Aunque tenemos una larga historia de actividades extractivas, las inversiones en el sector aumentaron rápidamente a partir de principios de la década de 1990, donde la minería ha logrado su mayor expansión en el territorio, con presencia no solo en la Costa y Sierra, sino también en la Selva, lo que ha contribuido posteriormente a grandes utilidades; Sin embargo, hay una limitada evidencia de que la minería haya contribuido al desarrollo local sostenible en el Perú, siendo otra causa que ha generado creciente conflictividad social (Bebbington, 2007b).

Los proyectos mineros vinculados a la tipología socioambiental, afrontan un problema que los hace más vulnerables que otros sectores industriales: están atados a un determinado espacio geográfico, es decir, no pueden trasladarse de lugar cuando las cosas no salen bien y deben encontrar una forma de convivencia con la población en las zonas de su intervención (Bebbington, 2007a), lo que implica proponer estrategias para la gestión social.

Esta tipología de conflicto social como proceso complejo en el cual sectores de la sociedad, el Estado y las empresas perciben que sus objetivos, intereses, valores o necesidades son contradictorios y esa contradicción puede derivar en violencia (Defensoria del Pueblo. Adjuntia para la Prevención de Conflictos Sociales y la Gobernabilidad, 2020). Afecta la funcionalidad del sistema social, ya que introduce la entropía generando problemas de integración sistémica (Alfaro Vargas \& Cruz Rodríguez, 2013).

Por otra parte, las estadísticas indican que, al menos durante la última década, el crecimiento y la estabilidad macroeconómica en el Perú han venido siendo sostenidos. El promedio del crecimiento económico ha sido de entre $7,5 \%$ a $8 \%$ anual. Luego de cuarenta años, el país ha logrado alcanzar superávits fiscales. El despegue económico no ha tenido su necesario correlato con los persistentes índices de pobreza, pobreza extrema y desigualdad, es más se acentuó en las áreas rurales de sierra y selva (Bedoya, 2014), lo cual ha contribuido a ser otro causal de conflictividad socio ambiental.

Y sobre los impactos generados sobre el medio ambiente, se vincula que las comunidades que se encuentran aledañas a una operación minera, solo la presencia de la unidad minera, puede significar correr peligro inminente a que sus recursos naturales como agua y suelo se contaminen (Castro, 2011). 


\section{Conclusiones}

Se ha verificado que las causas que impulsan los conflictos socioambientales son la calidad del agua, el temor a contaminación ambiental, la afectación a la biodiversidad, el uso superficial de tierras a favor de la empresa, el manejo de residuos sólidos debido a los impactos ambientales y a la salud de las personas, la falta de compromisos de responsabilidad social y los impactos generados en el medio ambiente.

Así mismo, aunque tenemos una larga historia de actividades extractivas en nuestro país, incrementándose a partir de principios de la década de 1990, donde las inversiones en el sector aumentaron rápidamente, logrando expandirse en el territorio, con presencia no solo en la Costa y Sierra, sino también en la Selva, lo que ha contribuido posteriormente al crecimiento y la estabilidad macroeconómica; sin embargo, paralelo a ello se ha incrementado los casos de conflictividad social en todo el país.

\section{Recomendaciones}

¿Qué hacer con los conflictos socioambientales? ¿Qué hacer con la dinámica conflictiva cuando viene acumula ndo patrones destructivos como profunda desconfianza, polarización y eventualmente violencia? ¿Cómo se puede actuar para canalizar constructivamente la energía del conflicto? Estas son algunas de las preguntas que se formulan permanentemente aquellos que están involucrados en el campo de la resolución de conflictos sociales.

Las respuestas a estas interrogantes forman parte de la implementación de mecanismos para intervenir los conflictos sociales, con el constante monitoreo de los casos latentes y activos, así mismo gestionar las causas subyacentes en la generación de conflictividad socioambiental, en su etapa de escalamiento, con el fin de evitar enfrentamiento entre las partes.

Entre los mecanismos de intervención que se debe implementar, es la prevención del conflicto, que se caracteriza por una intervención temprana en escenarios reales o potenciales de conflicto por medio de medidas que contribuyen a evitar comportamientos violentos. Se centra, en las reales dimensiones de los conflictos sociales y anticiparse a ellos para propiciar el surgimiento de procesos de cambio constructivo que beneficien a los directamente involucrados.

Otro enfoque, es la gestión del conflicto, donde se busca impactar rápidamente en el comportamiento de los actores mediante el dialogo, con el fin de llegar a posibles acuerdos entre las partes. 


\section{Referencias bibliográficas}

Alfaro Vargas, R., \& Cruz Rodríguez, O. (2013). Teoría del conflicto social y posmodernidad. R evista de Ciencias Sociales, II-III(128-129), 63-70. https://doi.org/10.15517/rcs.v0i128-129.8732

Barrantes, R., Tanaka, M., Vera, S., \& Pérez-León, M. (2010). El boom de los recursos naturales y las coaliciones presupuestarias: una ilustración con el caso peruano. Instituto de Estudios Peruanos, 46. Retrieved from http://scholar.google.es/scholar?hl=es\&q=\% $22 \mathrm{El}+$ boom+de+los+recursos+naturales $+\mathrm{y}+\mathrm{l}$ as+coaliciones+presupuestarias $\% 22+\& b t n G$ $=\& \mid r=\# 0$

Bebbington, A. (2007a). Elementos para una ecología política de los movimientos sociales y el desarrollo territorial en zonas mineras. In Instituto de Estudios Peruanos (Ed.), Minería, Movimientos Sociales y respuestas campesinas:una ecología política de transformaciones territoriales (1ra ed.). https://doi.org/10.2307/j.ctvn5tzhd.5

Bebbington, A. (2007b). Minería y desarrollo en el $P$ erú: con especial referencia al Proyecto Río Blanco, Piura (1ra ed.; IEP; OXFAM INTERNACIONAL; CIPCA; Peru Support Group, Ed.). Lima, Perú.

Bedoya, C. (2014). Evolución y tendencias de los conflictos sociales y ambientales en el Perú: Una lectura general. In P. y R. de C. ProDiálogo (Ed.), Minería, conflicto social y diálogo (1ra ed., pp. 27-41). Lima.

Bury, J. (2007). Neoliberalismo, minería y cambios rurales en Cajamarca. In Instituto de Estudios Peruanos (Ed.), Minería, movimientos sociales y respuestas campesinas. Una ecología política de transformaciones territoriales. (1 ra ed., pp. 49-80). Lima.

Castro, S. A. (2011). Pobreza y conflictos socioambientales, el caso de la mineria en el Perú. Pontifia Universidad Católica del Perú, Lima, Perú.

Defensoria del Pueblo. Adjuntia para la Prevención de Conflictos Sociales y la Gobernabilidad. (2020). Reporte de Conflictos Sociales (Vol. 15170). Lima.

Echave, J. De, Diez, A., Huber, L., Revesz, B., Ricard, X., \& Tanaka, M. (2009). Mineria y conflicto social (1 ra ed.; C. C. C. IEP, Ed.). Lima, Perú.

Flores Unzaga, C. (2016). Conviviendo con la minería en el Sur Andino (1ra ed.; Cooper Acción. Oxfan, Ed.). Lima, Perú.

Huamaní, G., Macassi, S., Alegría, J., \& Rojas, T. (2012). Hacia una mejor gestión de los conflictos socioambientales en el Perú (1ra ed.; Consorcio de Investigación Económica y Social, Ed.). Lima, Perú.

Mendoza, I. (n.d.). Conflictos sociales, medidas de solución y desigualdades: explorando algunas dimensiones. Retrieved from https://www.desco.org.pe/recursos/site/files/ CONTENIDO/1117/12_MendozaV_PH_dic1 6.pdf
Pinto, V. (2009). Reestructuración neoliberal del Estado peruano, industrias extractivas y derechos sobre el territorio. In CooperAcción - Acción Solidaria para el Desarrollo (Ed.), Minería y territorio en el perú. Conflicto, resistencia y propuestas en tiempos de globalización (1ra ed., p. 432). Lima, Perú.

Tanaka, M., Huber, L., Revesz, B., Diez, A., Ricard, X., \& Echave, J. de. (2007). Minería y conflicto social. Economia y Sociedad, 65, 7-17. 